\title{
The Embedded Research Librarian: A Project Partner
}

\section{Romain Féret}

Lille University Library

romain.feret@univ-lille.fr, orcid.org/0000-0002-1527-1482

\section{Marie Cros}

Lille University

marie.cros@univ-lille.fr, orcid.org/0000-0002-9744-9810

\begin{abstract}
This paper presents new services developed by the Lille University Library for European and National research project coordinators. This is a specific audience that libraries are not used to target, with a widely recognised institutional status and academic background. Supporting them in their coordination activities is an opportunity to gain a new role for libraries, which starts from the design of research at the submission stage and lasts several years after, during the project lifetime. These services help coordinators to meet their funders' expectations on Open Access and research data management. It is also a way to develop new collaborations with research units and some university services, such as the Grant Office. The Lille University Library has already supported the writing of forty grant proposals since 2017, including about thirty since early 2019. The Library currently follows twelve projects on Open Access, research data management or both. This second figure is likely to increase in 2020 due to the number of projects supported at submission stage since the beginning of 2019. The paper describes our set of services and the lessons we learned from our approach.
\end{abstract}

Keywords: open science; funder mandates; open access; research data management; library services 


\section{Introduction}

The University of Lille is a multidisciplinary university with 3,300 researchers and 66 research units. For several years, the Lille University Library has been developing an ambitious set of support services for researchers. This article presents services ${ }^{1}$ dedicated to research projects funded by the European Horizon $2020^{2}$ framework programme (H2020), or by the French Agency for Research $^{3}$ (ANR). We introduce the history of our approach before presenting the three main components of this set of services and their implementation. We emphasize that this approach allowed us to develop a deeper relationship with researchers with whom we were not so used to work before. Finally, we will draw some lessons from our experience and expose new challenges we could face in the future.

\section{From will to Action}

\subsection{H2020 Projects, an Open Door for Open Science?}

The Library of Lille started to work on Open Access to publications in 2012 and on research data management in 2013, but it is not until autumn 2016 that we have developed a strategy to meet the specific needs of research projects coordinators. Due to the H2020 Mandate on Open Access and to the Open Research Data Pilot, ${ }^{4}$ European projects seemed to be a good testing ground for supporting researchers with their research data management practices. We contacted our Grant Office in order to identify projects we could work with. However, we quickly discovered that at the beginning of 2017, all the projects coordinated by our University and covered by the first phase of the H2020 Open Data Pilot (2014-2017) had chosen to opt-out, which means they had no obligations regarding data management. The University of Lille was a partner in projects covered by the Open Research Data Pilot, but in those cases, the responsibility of data management fell on the coordinators of other institutions.

\subsection{Open Access, a Good Start}

It proved to be complicated to work with projects in which our researchers were only partners and whose influence on project management was limited. Since no project led by a researcher of our University was part of the Open 
Research Data Pilot, we diverted focus to projects subject to the Mandate for Open Access to Publications. In autumn 2017, we had our first meetings with two project coordinators: one ERC Advanced Grant ${ }^{5}$ beneficiary and a multibeneficiary H2020 coordinator. They were only partly aware of their Open Access requirements. Both thought that publications had to be published in Open Access on the publishers' platform. Due to the number of their project publications and to the cost of articles processing charges, they published only a selection of their articles in Open Access. Through several interviews with them, we identified their needs and how we could support them to improve their level of compliance with their Open Access obligations. We helped them to spot the publications they had to disseminate in Open Access and we explained them how they could do it into our institutional repository. Some of the publications did not contain the right acknowledgment of the European funding, which made dissemination reports inconsistent with the list of the project publications. We supported them at correcting this.

In return, we learned how research projects work from inside. We gained insight into the European Participant Portal, ${ }^{6}$ an online tool for coordinators and partners to monitor project progress and deliverables status. The Participants Portal is also used by the European Commission's Project Officers to interact with coordinators on the many mandates a grant agreement includes. Each project has an EC Project Officer, whose role is to support and monitor the project progress from start to end. We could also better understand the structure of European projects, and the delegation of partner responsibilities in work packages and deliverables, which proved very useful for making library services indispensable to grant applicants.

They had no objection against disseminating their publications in Open Access but they saw it as one more thing to do on a very long to-do list. From these talks, we understood that, through the Participant Portal, there was a strong link between the overall monitoring activities and the Open Access dissemination of publications. We obtained an access to their project on the EC Participant Portal which allowed us to help them with their Open Access obligations.

\subsection{When the Door Remains Shut}

At the end of 2017, the first H2020 project coordinated by a researcher of our university and covered by the Open Research Data Pilot started. We 
expected this project to be the first we could work with on research data management. However, when meeting the coordinator, we discovered that a consulting firm had been included in the project at the grant writing phase and was paid to perform all the tasks to follow-up with the progress of the project, which included monitoring Open Access to publications and the writing of the data management plan. We already knew that such stakeholders were involved at the grant writing phase but we did not expect them to be involved in the post-award phase of the project, which proved to be a naive thought.

The involvement of external consultants in monitoring Open Access compliance offered a direct competition to the Lille University Library. Although we were already aware of the importance of being involved at the pre-award submission, it crystalized the urgency to develop a new set of services, which cover both pre-award grant writing and monitoring of compliance in post-award phase. This proved to be a challenge, as most European projects worked with external consulting firms in the pre-award writing phase, few considered the Library as a local partner to help them address the recent mandates on publications and data. This forced us to look elsewhere.

In March 2018, the two H2020 coordinators we were working with on Open Access both planned to submit a project to the French National funder, the ANR. We analyzed the ANR grant application template to determine which sections the library could contribute to, such as: the impact section, the work package on project management, the institutional support to the project (IT infrastructure) or the risk analysis related to data management. We managed to convince them to add an Open Science dimension to their project, even though ANR did not have any obligations of this kind at that time. We helped two other coordinators to write their proposal during this call for project. A month later, a researcher who was drafting an ERC Starting Grant ${ }^{7}$ proposal asked for our help, after he had heard of the service we were delivering. The door was finally fully opened, which allowed us to implement a new set of services.

\section{Implementing a New Set of Services}

Our set of services can be divided in three sections, all linked but independent. 


\subsection{Grant Proposal Writing}

The support to grant proposal writing is offered to H2020 and ANR projects. We try to make sure the coordinators integrate Open Science throughout their projects, from research objectives to societal impact, dissemination and re-use of results, as well as commitment of adequate resources for implementation. We offer the coordinators the opportunity to show to the reviewers that they are fully aware of their Open Science obligations and that they will receive support from their institution on this matter. The paper "Winning Horizon 2020 with Open Science" (Grigorov, Elbæk, Rettberg, \& Davidson, 2015) was highly inspirational on identifying aspects that could be added to projects to improve their Open Science approach. Later, "Open Science in practice in FP9" brought new ideas we could work on (OpenAIRE, 2018).

Open access to publications impacts mainly the dissemination section but research data management planning has more diverse impacts, e.g. on ethics, on the project management work package or on the share of roles and responsibilities between partners. Discussing these aspects as early as the pre-award grant writing phase is far from being unnecessary since we have met several examples of projects in which conflicts arose because partners could not agree on the level of data sharing or on the tools to share and manage data.

Whenever contacted by researchers, we ask them to send us their proposal, even as an incomplete draft. Then, we arrange a meeting with them to discuss their project in order to know which level of openness they would like to go to and which kind of strategy they could establish to achieve it. We also ask questions on different aspects related to Open Access and data management. This interview is conducted in a rather systematic way but with flexibility to accommodate project needs. On the one hand, we try to identify specific issues that could cause difficulties during the project or at the submission stage, such as: big volume of data, sensitivity of data, different culture of data sharing between partners and we help the researcher to make the way these issues will be dealt with more explicit. On the other hand, we spot what could be showcased with data management, such as the constitution of very interesting data sets or an ambitious data sharing policy. We also help them to identify other university services they should go to, such as the Data Protection Officer or the IT services. 


\subsection{Monitoring Compliance with Open Access Requirements}

Our Library acts as an "Open Access manager" for the projects. Our role is to make sure all the project publications are Open Access and well reported on the participant portal. The regular workflow is that researchers upload their publication in a repository as soon as it is published, with an embargo when necessary, and they send us an email with the repository link. It enables us to make sure that the paper is well harvested by OpenAIRE. Then we correct metadata on the participant portal and validate the publication. It often occurs that publications are not uploaded in Open Access or that we are not informed of the publication of a paper. To avoid this blind spot, we set up an alert for each project in the Web of Science and in Scopus by using its Cordis number. We regularly inform project coordinators that publications of their partners acknowledge their project while they have not heard about it. We take part in project meetings to train all the partners on Open Access. When one of their publication is not in Open Access we inform them on how they can make it open. When a project publication has been uploaded into a repository without the project metadata, we contact the repository manager to add it. Finally, we may be in contact with the project officers to answer their questions on Open Access.

\subsection{Supporting Research Data Management}

Support to research data management is the component of the set of services on which we are less experienced since few H2020 projects are coordinated by researchers of our University and because the ANR has set up a data management policy only for projects funded since 2019. We currently follow two ANR projects funded in 2018, which committed to draft a data management plan, though they did not have to do so, and we supported two individual H2020 projects to write their data management plan (one ERCAdvanced and one Marie Curie Individual Fellowship). Though research data is a more and more commonly discussed topic, researchers are still quite reluctant to formalize their practices and not at ease with writing them down. Data management plan (DMP) writing is still considered by some researchers as a loss of time to produce a document that seems to make more sense for the funder than for the project consortium. Trust-building and establishing sound relationships at the pre-award grant writing phase and the support on 
monitoring the compliance with Open Access requirements help us to overcome this reluctance.

To help the project team to initiate the process of writing their data management plan, we write an embryonic version based on the information collected at the submission stage and we perform one more interview at the beginning of the project to complete the information and to correct possible misunderstandings. We also try to identify a member of the project with capacity for data management and who has more time than the coordinator to spend on writing the DMP and ensuring the implementation of good practices. We present them the DMP as a short, operational and living document and we endeavour to identify small and concrete actions that can initiate a change in practices. For instance, we propose that researchers upload a dataset into a repository with underlying data of each of the project publication, and open up the metadata even if they keep the datasets with time-specified embargoed access. It is an easy way to make them describe their datasets and preserve their data on public and secured infrastructures. This pragmatic approach is quite successful. The ERC-Advanced researcher we provide support to, has already uploaded two datasets in Zenodo since the beginning of October 2019, while he had never done it before. ${ }^{8}$ He now plans to upload systematically a dataset in Zenodo for each publication related to his ERC project.

\subsection{A Set of Services Expanding Quickly}

As shown in Figure 1, we first implemented these services on the campus of Sciences and Technology as an experimentation, from 2016. In January 2018, the merging of the three previous universities of Lille, which had different disciplinary scopes, created the University of Lille. In May 2018, we started to support coordinators on the Humanities and Social Sciences (HSS) campus. We waited until the beginning of 2019 to work with projects on the Health Sciences campus.

Once merged, the University of Lille brings together more than 3,300 researchers, spread over four campuses. Most of the European and National projects are distributed between the Sciences and Technology campus and the Health Sciences campus. Few projects are conducted on the HSS campus and even less on the Law campus. As shown in Figure 2, we are helping 
Fig. 1: Timeline of the extension of the support of H2020 and ANR projects by the Library.

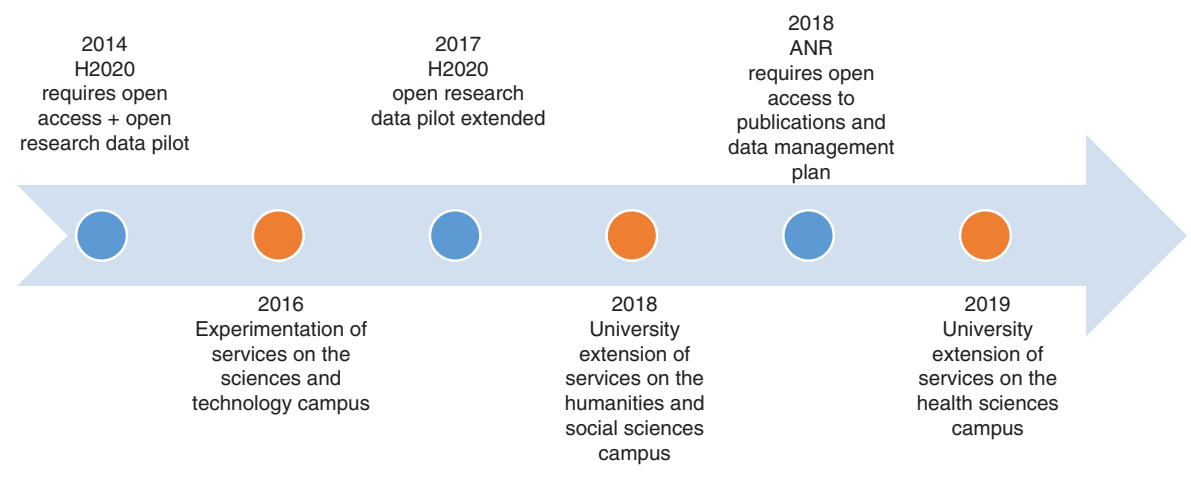

a growing number of researchers when writing a grant proposal. The new requirements adopted by the ANR in 2019 explain the important increase in the number of projects supported during the $1^{\text {st }}$ semester of this year. So far, about two thirds of the supported projects have been applying for an ANR grant and one third for a H2020 funding.

The number of ongoing projects we are following is also increasing (see Figure 3), but at a slower pace and with a lag compared to the number of projects helped that received bidding assistance, as it takes several months between the submission date and the starting date of projects. For

Fig. 2: Number of researchers supported for the writing of their grant proposal ( $1^{\text {st }}$ of December 2019).

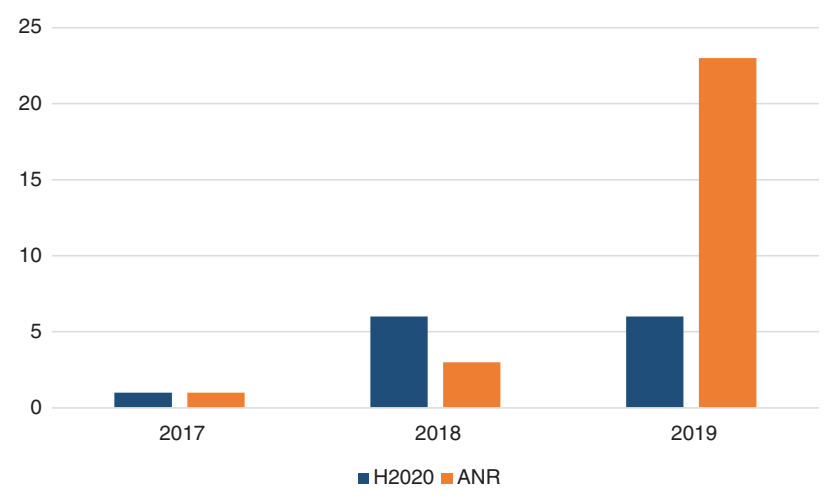


example, most of the ANR grants proposals were submitted in March 2019. Coordinators will be informed of acceptance or rejection in July and the selected projects will start between October and April 2020, which gives the time to the Library to anticipate the projects' needs. Considering the increase in the number of projects helped at the submission stage in 2019, it is very likely that the number of ongoing projects supported will increase from autumn 2019.

We support ANR ongoing projects on an individual basis only when we have supported them at writing their grant proposals. For ANR projects that we have not supported at writing their grant application, we offer collective training sessions on data management and Open Access and we review their DMPs before they submit it to their funder. Three workshops on the writing of a DMP took place in our libraries in November 2019. Twenty-eight researchers representing twenty-three research projects took part to one of these training sessions. They are not counted in Figure 3.

So far, we have been working primarily with projects on the Science and Technology campus (see Table 1), but we hope to help more and more Health sciences coordinators. In our university, the number of researchers participating in ANR projects is much higher than the number of researchers who take part in H2020 projects. The new requirements on Open Science of the ANR should be a good opportunity to reach a wider audience in these disciplines.

Fig. 3: Number of ongoing projects supported ( $1^{\text {st }}$ of December 2019).

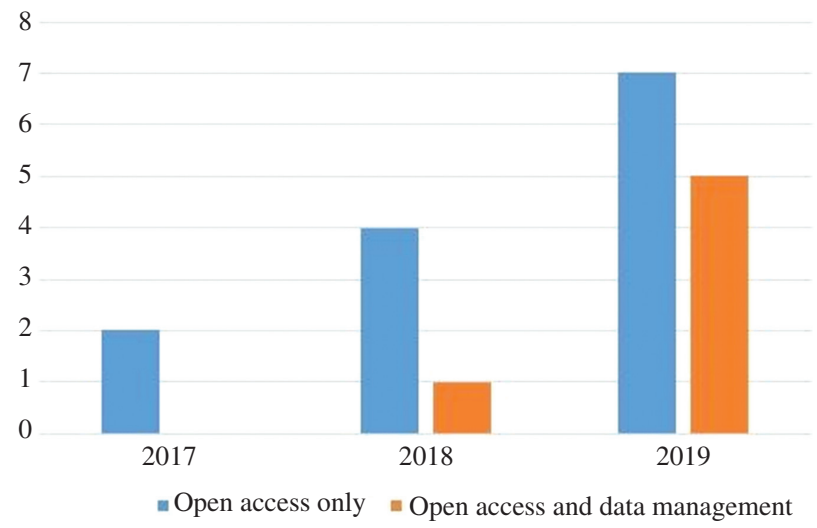


Table 1: Disciplinary distribution of projects supported (1 $1^{\text {st }}$ of December 2019).

\begin{tabular}{lll}
\hline & Pre-award submission & Post-award phase \\
\hline Sciences and Technology & 24 & 7 \\
Health Sciences & 3 & 3 \\
Humanities and Social Sciences & 11 & 1 \\
Multi-disciplinary & 2 & 1 \\
Total & $\mathbf{4 0}$ & $\mathbf{1 2}$ \\
\hline
\end{tabular}

\subsection{Impact of the Library Support}

The impact of the library support on grant applications cannot be measured directly from the success rate of projects, as the projects are very different from each other and the normal success rate is quite low for French and European projects (between 15 and 20\%) with a wide variety of factors that make a project funded or not. The dissemination strategy and research data management are only a small part of the proposals. To obtain statistically significant results, equivalent project samples should be compared for factors such as: discipline, call for projects, quality of the coordinator $\mathrm{CV}$ and of the consortium. Our impact can be measured more qualitatively by the quality of the sections to which we have contributed and through the positive evaluation that these projects have received about their open science strategy. About half of the evaluation reports we have received to date mention a good dissemination strategy and an interesting Open Access approach (see box for examples). Evaluators in their report do not yet consider much the research data management strategy.

"The proposed strategy for dissemination [...] is convincing and efficient" Source: ANR Proposal Evaluations, 2018

"The dissemination strategy is well established: scientific publications, deposit on institutional platform, genomic data publications in ENA (European Nucleotide Archive)."

Source: ANR Proposal Evaluations, 2019 
"The importance for this research is also reflected in the dissemination plan. The project receives a strong support from the Lille university library whose $\mathrm{H} 2020$ dedicated service will make sure all the peerreviewed publications from the project are made open access"

Source: H2020 Evaluation Summary Report, 2018

The impact of the library on compliance with Open Access obligations is easier to highlight. We currently support seven H2020 projects that are required to publish their publications in Open Access ${ }^{9}$. The oldest started in 2015 and the most recent one in 2019.

Looking for these projects from their Cordis number in the Web of Science (30 ${ }^{\text {th }}$ of November 2019), we found 106 publications related to these projects. We checked these publications with the project coordinators. In fact, seven of these publications were not part of these projects. Out of the 99 remaining publications, 95 are Open Access (96\%) and 93 are available into an Open Access repository (94\%) which is an excellent compliance rate with the H2020 Open Access policy, higher than the average compliance rate. ${ }^{10}$

Recent research on researchers' compliance with Open Access obligations has shown that obligations are usually not enough to ensure Open Access to publications (Larivière \& Sugimoto, 2018; Vincent-Lamarre, Boivin, Gargouri, Larivière, \& Harnad, 2016). In the study of Larivière and Sugimoto, 73\% of ERC projects publications are in Open Access and 67\% of publications are in green Open Access. In 2018, a note on Open Access to publications produced by H2020 projects provided quite similar figures for the period 2014-2017. Based on OpenAIRE data, the Open Access rate was of $63.2 \%$ and less than $50 \%$ of publications were in green Open Access, while the Open Access rate was of $80.6 \%$ based on the data provided by the internal European portal, with about $56 \%$ of the publications in green Open Access (European Commission - DGRI, 2018). The report authors precise that the data coming from the European portal is self-reported by researchers and could be a bit overestimated.

Our support for projects with data management obligations is too recent to have meaningful indicators. We intend to evaluate the quality of the data management plan of the researchers supported. The number of data sets disseminated over the life span of the project will be a good indicator of the 
actual implementation of good research data management practices, beyond simply writing a DMP. In the future, we would also like to keep track of the Open Science costs budgeted in the grant proposals and of the actual spending on Open Science activities.

\section{A New Role for Librarians}

The implementation of this new set of services allows us to build up a new kind of relationship with researchers who were not used to working with us before and to develop new collaborations.

\subsection{A New Public}

One of the most striking inputs of our approach is our ability to reach a new kind of researcher. So far, when we were in contact with experienced researchers it was mainly because they were research unit directors or Open Science activists. Some figures on the coordinators of the $8 \mathrm{H} 2020$ ongoing projects which we work with show that we reach high level researchers, often considered leaders in their field. They are aged between 36 and 61 years (average 52 years) while we usually work with young researchers or PhD students. Based on Scopus data (12/12/2019), they have published an average of 276 publications, with an average h-index of 49 (from 8 to 132). Altogether, their works have received a number of citations in Scopus that exceeds 105,000 (average of 13,200) and they have collaborated with an average of more than 700 co-authors. Finally, six of these researchers are research team leaders. The limitations of bibliometric data are well known, but these mere figures show that we reach researchers with important scientific responsibilities. This has several positive effects on the Library perception by other researchers. This gives us legitimacy and it brings us mid-career researchers. We recently launched our institutional repository LillOA, ${ }^{11}$ connected to the French National repository HAL ${ }^{12}$. That some ERC-advanced researchers upload their publications into our repository is a strong incentive for other researchers.

\subsection{A New Perception of the Librarian's Role}

Grant proposal drafting is an important and stressful task for researchers. Taking part in it is very rewarding for librarians, especially when the 
collaboration explicitly makes positive impact on the proposal evaluation. This is the basis for a new relationship between librarians and researchers. Within the research teams, we have a recognized role of Open Access or open science manager, which acknowledges our participation in the project and makes us identifiable by the coordinator and its partners. Our help is well received since we do not introduce new requirements but we help to meet existing ones and to improve the progress of the projects. All this is independent of the benefits of winning the proposals. Converting proposals to grants is a concrete measure of success, but even if the proposals are not funded, the design of a research project is an opportunity to build a strong relationship between librarians and researchers. Our service is based on a reiterative process involving many unfunded proposals, with the evaluation process providing very interesting information to improve our approach.

When the proposals are funded, relations with project coordinators also differ from exchanges with other researchers because of their duration. From submission to the end of the project, we may be in contact with a coordinator regularly for more than 5 or 6 years. As most coordinators regularly submit projects, we also hope to work with them on new projects in the future. For instance, we have already twice helped a researcher write a grant proposal. One of them was accepted and we are working with her on a third project.

\subsection{New Collaborations}

The development of these services has been made possible by a cooperation with various stakeholders on our campus. The first of them is our university Grant Office which deals with the administrative and financial part of grant proposals. We meet regularly to ensure a smooth support to projects. We also take part in information sessions they organize for project coordinators. The vast majority of the 66 research units of our university also depends on other research organizations such as the French National Centre for Scientific Research (CNRS), the university hospital and other local or national research organizations. These organizations have often their own grant office and a convention states how the projects are distributed between them. Our Library assists all researchers in our university research units, regardless of the organization responsible for its management. 
We also interact with other stakeholders of data management such as the data protection officer (DPO) or the IT services. The DPO can bring his expertise when a project plans to collect or process personal data. H2020 project coordinators have to submit a self-ethics assessment at the bid stage. This is a good tool to explore issues related to sensitive data. As this document is only an administrative appendix and is not evaluated by the reviewers, we ensure that the coordinators accurately describe how they will handle this kind of data in the main part of their project, so that the reviewers do not doubt the project's feasibility. Although ANR projects are not required to draft a specific ethics document, these elements may be explained in the section on risk management. A common approach enables to ensure consistency between the ethics section and the data management and dissemination strategy. For its part, the information technology service provides the secured infrastructures to store the data during the project and for a couple of years after its end. An estimation of the project's data storage needs is important as soon as the proposal is drafted, to find solutions that meet those needs and to budget associated resources, as appropriate. Finally, the research and innovation service can help to find a balanced strategy between dissemination and economic promotion of some specific data such as software, or for the sharing of data ownership between partners. However, at the moment, the establishment of this strategy is usually postponed till after the beginning of the project, when the consortium agreement is written.

Research unit directors are a good contact point to reach project coordinators since they know which researchers are often applying for funding. In some research units, there is a grant officer with whom we can be in contact. So far, we have been specifically working with 7 research units in which we are identified as a go-to service to take Open Science into account in writing grant proposals. We met with the research unit directors or with the grant officer and we made a presentation of our set of services to these units' researchers. About two thirds of the projects we work with come from one of these units, which shows it is a relevant way to reach our public, while other researchers have heard of our service mainly through the communication of our university Grant Office.

\subsection{New Skills for Librarians}

Being integrated into research projects has been a good opportunity to develop new skills in writing grant proposals, such as defining an Open Science strategy, identifying risks related to data management in various 
disciplines, knowledge of the rules of eligibility for funding, the compatibility between resources and objectives. We also learned a lot about how to manage research projects, how to inform and train partners with diverse interests and with different implications in a project (coordinator, work package leaders, other researchers). We now have a better understanding of the language of funders and how research projects are assessed by reviewers and monitored by project officers. We also developed new partnerships in our own institution with the Grant Office and other staff in research units and learned how to use funders' tools such as the OpenAIRE services, the Participant Portal or the Cordis database. Ultimately, by pro-actively engaging all lifecycle actors in research grants, we also have the collective opportunity to change the perception of research librarians, and to make the "research librarians become an indispensable partner in optimally disclosing the diverse outputs of the research process" (Ekstrøm, Elbæk, Erdmann, \& Grigorov, 2016).

\section{Learnt Lessons and Perspectives}

Our experience allows us to identify four main lessons that could help colleagues who would like to develop such a set of services in their own library.

\subsection{Be Proactive}

The first lesson is that working with research projects requires librarians to be proactive. Indeed, at the beginning we were not identified at all as a possible stakeholder of grant proposal writing or of project management. Our cooperation with our Grant Office and our collaboration with project coordinators finally allowed us to become a recognized player in project management. Researchers will not go to their library to ask for help in carrying out their project unless you told them what you could do for them. As already mentioned, writing a grant application is often a stressful period when many researchers and services have to work together on the same document. It is important to be identified by coordinators as one of them as soon as they start working on it. Initially, we expected the Grant Office to connect us with the coordinators but grant officers are often contacted very late by coordinators, sometimes just a couple of days before the submission date. It is therefore wise to have direct relationships with research units to be involved in projects earlier. 


\subsection{Do take your Place}

When we work with project coordinators, we try to build a balanced relationship with them, based on mutual trust and reciprocity. It is necessary to make sure that this relationship lasts for several years. At the preaward submission stage, we require that coordinators send us the final version of the proposal and all the reviews they receive, which allows us to improve our work. With ongoing projects, we ask coordinators to give us a role of Open Science manager. Though informal, this role gives us the legitimacy to interact with the partners and with the project officers. We also expect researchers to follow at least a part of our advices and to make their best to ensure that their project complies with their Open Science requirements. For example, if a coordinator did not disseminate its publications in Open Access, we would stop managing the continuous report of its publications on the Participant Portal. Fortunately, this situation has not happened so far.

\subsection{Do not take Open Access for Granted}

Complying with Open Access requirements may seem like an easy task that researchers should be aware of, since it has been an H2020 obligation for several years. However, experience has shown us that it is difficult for coordinators to carry out their project in all its dimensions since there are many requirements they should comply with. Reading the full version of the Annotated Model Grant Agreement (European Commission, 2017) is a good way to note that Open Science is only one among many dimensions of research project.

Multi-beneficiary H2020 projects are especially complicated to manage and many coordinators are not aware of all the publications linked to their project and they do not know Open Access enough to feel at ease to ask their partners to upload their publications into a repository. Tools for monitoring Open Access to publications such as OpenAIRE and the European Participant Portal work well, but there is still room for improvement to ensure a smooth workflow from uploading publications into repositories to their visibility on the Participant Portal, with correct metadata. So, Open Access should not be taken for granted and this approach is actually a very good way to start working with project coordinators. 


\subsection{Funder is the Key}

Regardless of the quality of service you provide to researchers, the opinion of funders will always be the most important. The evaluation reports received by the projects are very instructive on how reviewers considered the contribution on Open Science. It can differ a lot from one reviewer to the other, which is a good indicator of their Open Science level of sensibility. This sensibility can be expected to become increasingly important in the years to come. Showcasing good reports from reviewers is also an interesting way to convince other researchers to work with you. Open Science is not as developed in France as it is in other European countries and some of our researchers are still reluctant towards it. The fact that the research projects, even submitted to the French research funding body, are often examined by foreign researchers, proved very convincing. For ongoing projects, it is important to be involved in the exchanges about Open Science between the coordinator and the funder project officer. This is an opportunity to facilitate discussion and to provide accurate information on the level of openness of the project outputs.

\subsection{Perspectives and Challenges}

We have faced several challenges since the beginning of the implementation of this new approach that we have managed to overcome, but new challenges are coming. The number of supported researchers is increasing rapidly and will likely continue in the coming years. This is primarily due to the new requirements of the ANR, which covers a number of projects much higher than H2020 in our university. Then, projects often last from three to five years. With more and more new projects helped at the submission stage, new projects will add to old one every year for at least three or four years.

Until recently, these services have been developed with rather limited resources. At the beginning of 2017 it represented about 0.1 FTE and it increased little by little to about 1.2 FTE in September 2019, shared on three members of the staff. To scale these services up, it will be necessary to widen the number of colleagues involved. From January 2020, we plan to train more colleagues to take part in the monitoring of the compliance with Open Access requirements. We also intend to move from a project by project approach to a more collective one, with common training sessions for example. But, building a strong relationship with coordinators will remain crucial. It is also 
necessary that projects rely more and more on their own resources to meet their needs on research data management.

\section{Conclusion}

Funders' mandates are often considered as one of the most important incentives to develop Open Science. This article presents the services dedicated to project coordinators developed by the Lille University Library. These services can be grouped in three components: help writing grant proposals, support to disseminate publications in Open Access and to manage research data of ongoing projects. It has proven to be a very efficient way to improve projects' compliance with Open Science obligations and to help them manage their project. Helping projects from the submission stage opens up many opportunities for libraries, because taking part in projects allows librarians to work with researchers from the design of their research and reach researchers who are leaders in their field. It is also an opportunity to gain new skills and to collaborate with other university services.

\section{Acknowledgment}

We thank Ivo Grigorov for the stimulating discussions on the support that librarians can offer to researchers when writing their grant applications and for his insightful comments on the draft manuscript.

\section{Data availability}

The data about the publications from the $\mathrm{H} 2020$ projects will be made available in Zenodo: Féret, Romain \& Cros, Marie (2019, December). Research data supporting for "The embedded research librarian: a project partner." Zenodo. http://doi.org/10.5281/zenodo.3564521.

\section{References}

Ekstrøm, J., Elbaek, M., Erdmann, C., \& Grigorov, I. (2016). The research librarian of the future: Data scientist and co-investigator. LSE Impact of Social Sciences. [Blog]. Retrieved 
May 15, 2019, from: https://blogs.lse.ac.uk/impactofsocialsciences/2016/12/14/ the-research-librarian-of-the-future-data-scientist-and-co-investigator $/$.

European Commission. (2017). H2020 Programme - Annotated model grant agreement. Version 4.1. Retrieved May 15, 2019, from: https:/ / ec.europa.eu/research/ participants/data/ref/h2020/grants manual/amga/h2020-amga en.pdf.

European Commission. Directorate-General for Research \& Innovation. (2018). Background information note. Open Access to scientific publications produced with Horizon 2020 funding: State of play in 2017. Retrieved

November, 29, 2019, from: http://data.europa.eu/euodp/en/data/ dataset/open-access-to-scientific-publications-horizon2020/resource/ c8d2cef0-55e7-423a-88bd-f86c3099cb4d.

Grigorov, I., Elbæk, M., Rettberg, N., \& Davidson, J. (2015). Winning Horizon 2020 with open science. Retrieved May 13, 2019, from https://zenodo.org/record/12247.

OpenAIRE. (2018). Open science in practice in FP9. Retrieved May 18, 2019, from https://www.openaire.eu/openaire-fp9-pdf/download.

Larivière, V., \& Sugimoto, C.R. (2018). Do authors comply when funders enforce open access to research? Nature, 562, 483-486. https:/ / doi.org/10.1038/d41586-018-07101-w.

Vincent-Lamarre, P., Boivin, J., Gargouri, Y., Larivière, V., \& Harnad, S. (2016). Estimating open access mandate effectiveness: The MELIBEA score. Journal of the Association for Information Science and Technology, 67(11), 2815-2828. https://doi. org/10.1002/asi.23601.

\section{Notes}

${ }^{1}$ University of Lille Library, https:/ / lilliad.univ-lille.fr/en/
open-science-for-anr-and-h2020-projects.

${ }^{2}$ What is Horizon 2020, https:/ / ec.europa.eu/programmes/horizon2020/ what-horizon-2020.

${ }^{3}$ French Agency for Research, https://anr.fr/en/.

${ }^{4}$ H2020 Mandate on Open Access and Open Data Pilot, Grant Agreement Article 29, http:/ / ec.europa.eu/research/participants/data/ref/h2020/grants manual/amga/ h2020-amga en.pdf\#page $=242$.

${ }^{5}$ European Research Council Advanced Grants, https://erc.europa.eu/funding/ advanced-grants.

${ }^{6}$ European Commission Participants Portal, https://ec.europa.eu/info/ funding-tenders/opportunities/portal/screen/home. 
${ }^{7}$ ERC Starting Grants, https:/ / erc.europa.eu/funding/starting-grants.

${ }^{8}$ Idrissi, H., Samaee, V., Lumbeeck, G., van der Werf, T., Pardoen, T., Schryvers, D., \& Cordier, P. (2019). Supporting data for "In situ Quantitative Tensile Tests on Antigorite in a Transmission Electron Microscope." [Data set]. Zenodo. http://doi.org/10.5281/ zenodo.3515901 and Clitton Nzogang, B., Thieme, M., Mussi, A., Demouchy, S., \& Cordier, P. (2019). Research data supporting for "Characterization of recovery onset by subgrain and grain boundary migration in experimentally deformed polycrystalline olivine." [Data set]. Zenodo. http://doi.org/10.5281/zenodo.3492179.

${ }^{9}$ Acronyms and Cordis number of projects are (in order of start date): FAIR-PARK II (633190), FireBar (670747), PANG (690836), Immunobile (694717), TimeMan (787198), Zeocatalyst (795091) and PEARL (847568).

${ }^{10}$ The data on publications is available in Zenodo : Féret, Romain \& Cros, Marie.

(2019, December). Research data supporting for "The embedded research librarian: a project partner." Zenodo. http:// doi.org/10.5281/zenodo.3564521.

${ }^{11}$ Lille Open Archive, http://lilloa.univ-lille.fr/.

${ }^{12}$ HAL, https://hal.univ-lille.fr/. 